ISSN 1112-9867

Available online at

http://www.jfas.info

\title{
STUDY OF PERFORMANCES OF ORGANIC SOLAR CELLS BY ANALYSIS OF MAIN COMPONENTS
}

\author{
M. R Merad Boudia ${ }^{1}$, A. M. Ferouani ${ }^{1}$, A. Cheknane ${ }^{2}$ and B. Benyoucef ${ }^{1}$ \\ ${ }^{1}$ Université de Tlemcen, Faculté des sciences, U.R.M.E.R., B.P 119, 13000, Algérie \\ ${ }^{2}$ L.E.D.S.M.D. Université Amar Telidji de Laghouat Algerie
}

Received: 26 February 2011 / Accepted: 03 April 2010 / Published online: 30 June 2011

\begin{abstract}
The analysis of the data is currently, in the statisticians, the object of a true phenomenon of mode, characterized at the same time by the passion and the rejection. To determine the characteristics of a whole of data are obviously not an end in itself, but this lapse of memory is unfortunate because the type of data available always influences the direction of research. The factorial analysis in normalized principal components (PCA), is particularly adapted to the reduction of tables of measurements, where one generally works on a matrix of correlation between characters. In this article we will report our results of analysis of performances of organic solar cells by using what one call the datamining materials. All information was presented in the tables envisaged by an ACP method of the studied semiconductors. Factorial analyzes substitutes for primitive measurements of new variables or factors (which are linear combinations of the primitive variables) with the help of which it is thus possible to optimize the visualization of the data in a restricted number of figures. Large at the modo we present, the results related to the development of new approaches of Datamining, Analysis in principal components (ACP), of the data-processing techniques which were applied to organic semiconductors and combined with metals to predict new materials or best performing coatings.
\end{abstract}

Keywords: organic solar cells, gap energie, effiency, PCA

Author Correspondence, e-mail: mbmr25@yahoo.fr

ICID: 1020786 


\section{INTRODUCTION}

Les plastiques sont des composés carboniques formées d'une chaine de molécule (polymères) et/ou d'entités élémentaires (monomères). Les matières plastiques sont généralement utilisées parce qu'ils présentent un certain nombre d'avantages : légèreté, flexibilité et résistance contre l'influence de l'environnement telle que la corrosion. En outre, la raison la plus importante pour laquelle les matières plastiques sont souvent utilisées avec une variété d'applications est la facilité de leur fabrication, ce qui rend l'industrie des polymères conducteurs plutôt bon marché.
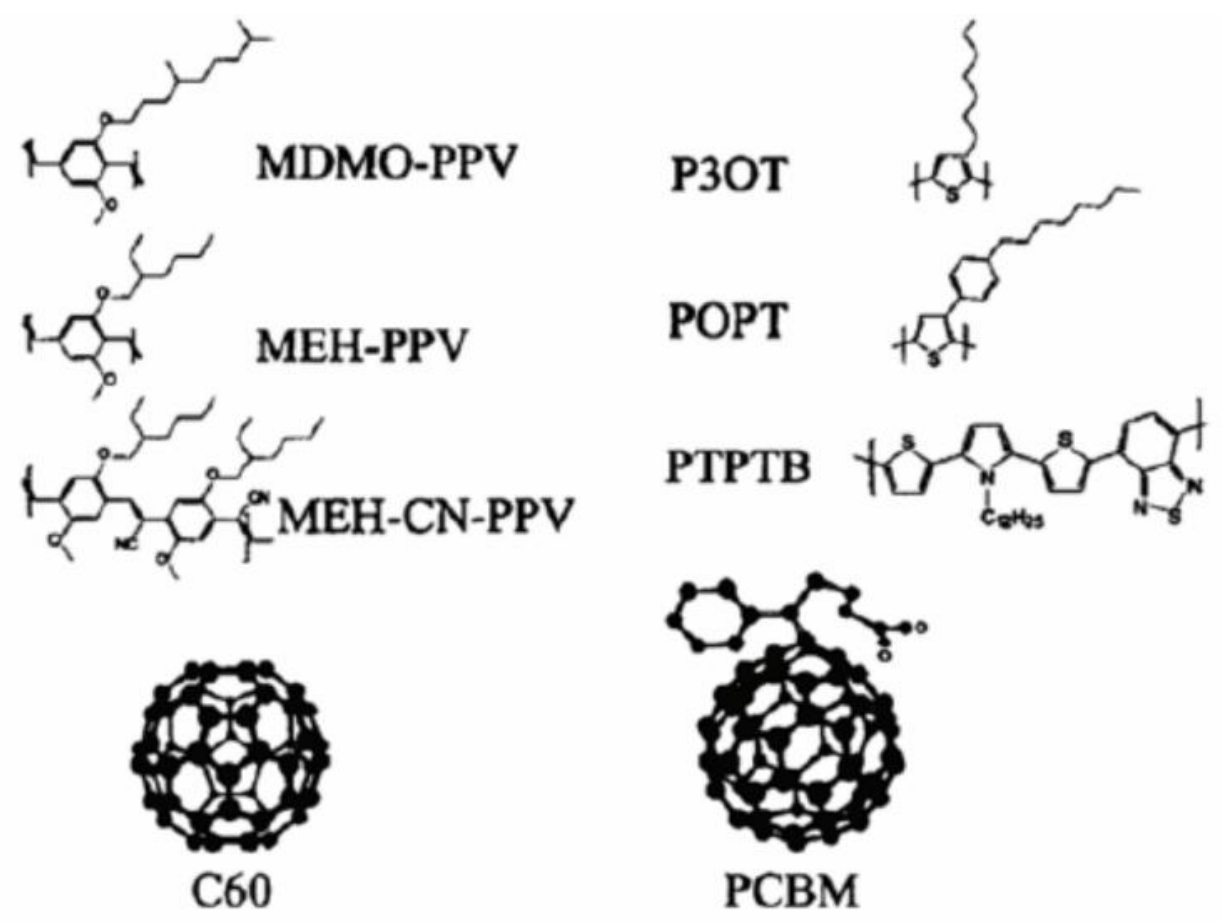

Fig.1. Les polymères semi-conducteurs ordinaires et les macromolécules utilisées dans les cellules photovoltaïques organiques

\section{ANALYSE EN COMPOSANTES PRINCIPALES (ACP)}

L'analyse en composantes principales ("Principal Component Analysis"), en abrégé $\mathrm{ACP}$, est une technique descriptive permettant d'étudier les relations qui existent entre les variables, sans tenir compte, a priori, d'une quelconque structure [2-3], son utilisation pour l'exploitation des données remonte au début du siècle dernier. Elle est principalement issue des travaux de psychologues américains [Pearson 1901], [Spearman 1904] [4-12].

L'ACP propose, à partir d'un tableau rectangulaire de données comportant les valeurs de $p$ variables quantitatives pour $n$ unités (appelées aussi individus), des représentations 
géométriques de ces unités et de ces variables. Ces données peuvent être issues d'une procédure d'échantillonnage ou bien de l'observation d'une population toute entière. Les représentations des individus permettent de voir s'il existe une structure, non connue a priori, sur cet ensemble d'individus [1]. De façon analogue, les représentations des variables permettent d'étudier les structures de liaisons linéaires sur l'ensemble des variables considérées. Ainsi, on cherchera si l'on peut distinguer des groupes dans l'ensemble des unités en regardant quelles sont les unités qui se ressemblent, celles qui se distinguent des autres, etc. Pour les variables, on cherchera quelles sont celles qui sont très corrélées entre elles, celles qui, au contraire ne sont pas corrélées aux autres, etc.

Le but de l' ACP est d'identifier la structure de dépendance entre des observations multivariables, afin d'obtenir une description ou une représentation compacte de ces dernières.

L'idée de base de l'ACP est de réduire la dimension de la matrice des données, en retenant le plus possible les variations présentes dans le jeu des données de départ. Cette réduction ne sera possible que si les variables initiales ne sont pas indépendantes et ont des coefficients de corrélation entre elles non nuls. Ces variables initiales sont transformées en de nouvelles variables, appelés composantes principales. Elles sont obtenues par combinaisons linéaires des précédentes et sont ordonnées et non corrélées entre elles [4].

L'analyse en composantes principales cherche à identifier les vecteurs propres orthonormaux et les valeurs propres de la matrice de dispersion des variables originelles. Les vecteurs propres orthonormaux sont utilisés pour construire les composantes principales et les valeurs propres sont les variances des composantes principales correspondantes [2]. 
Table 1. Nous avons appliqué l'ACP au résultats expérimentaux publiés dans la littérature par[13]

\begin{tabular}{|l|c|c|c|c|c|}
\hline $\begin{array}{c}\text { Dispositifs } \\
\text { Photovoltaïques }\end{array}$ & $\mathbf{E}_{\mathbf{g}}(\mathbf{D})[\mathbf{e V}]$ & $\mathbf{E g}(\mathbf{A})[\mathbf{e V}]$ & $\mathbf{V}_{\mathbf{o c}}[\mathbf{V}]$ & $\mathbf{e f f [ \% ]}$ & $\begin{array}{c}\mathbf{E}_{\mathbf{g}} \mathbf{-} \\
\mathbf{e V}\end{array} \mathbf{\text { oc }}[\mathbf{e V}]$ \\
\hline PCPDTBT:[70]PCBM & 1,38 & 1,7 & 0,62 & 5,45 & 0,76 \\
\hline PSIFDBT:[60]PCBM & 1,82 & 1,7 & 0,9 & 5,4 & 0,8 \\
\hline PSBTBT:[70]PCBM & 1,45 & 1,7 & 0,68 & 5,1 & 0,77 \\
\hline P3HT:[60]PCBM & 1,91 & 1,7 & 0,61 & 4,4 & 1,09 \\
\hline PF10TBT:[60]PCBM & 1,95 & 1,7 & 1 & 4,2 & 0,7 \\
\hline PBBTDPP2:[70]PCBM & 1,43 & 1,7 & 0,61 & 4 & 0.82 \\
\hline PCDTBT:[60]PCBM & 1,88 & 1,7 & 0,89 & 3,6 & 0,81 \\
\hline $\begin{array}{l}\text { MDMO- } \\
\text { PPV:[70]PCBM }\end{array}$ & 2,1 & 1,7 & 0,77 & 3 & 0,93 \\
\hline
\end{tabular}

\section{RESULTAT ET DISCUSSION}

Le problème posé est l'étude du comportement d'une population dont on dispose d'un échantillon formant un ensemble fini $\mathrm{I}$, vis à vis d'un ensemble fini $\mathrm{V}$ de variables numériques. L'ensemble des lignes du tableau des données est indexé par I de taille $\mathrm{n}$ et celui des colonnes par $\mathrm{V}$ de taille $\mathrm{m}, \mathrm{x}_{\mathrm{ij}}$ est la mesure de la $\mathrm{j}^{\text {ème }}$ variable $\mathrm{V}_{\mathrm{j}}$ sur le $\mathrm{i}^{\text {ème }}$ élément i de I. Ainsi donc, la distance entre deux éléments i et i' de I s'écrit [14] :

$$
d^{2}\left(i, i^{\prime}\right)=\sum_{1 \leq j \leq m}\left(X_{i, j}-X_{i, j}\right)
$$

Cela nous permet de faire une analyse en composantes principales (A.C.P.) et d'obtenir des résultats de la base de donnée[13] présentés sous forme de représentation graphique.

Tableau 1. Base de donnée des chalcopyrites ternaire II-IV-V 2.

Valeurs propres :

\begin{tabular}{lrrrr}
\hline & \multicolumn{1}{l}{ F1 } & \multicolumn{1}{l}{ F2 } & \multicolumn{1}{c}{ F3 } & \multicolumn{2}{l}{ F4 } \\
\hline Valeur propre & 2,122 & 1,230 & 0,520 & 0,128 \\
Variabilité (\%) & 53,044 & 30,739 & 13,006 & 3,210 \\
\% cumulé & 53,044 & 83,783 & 96,790 & 100,000 \\
\hline
\end{tabular}




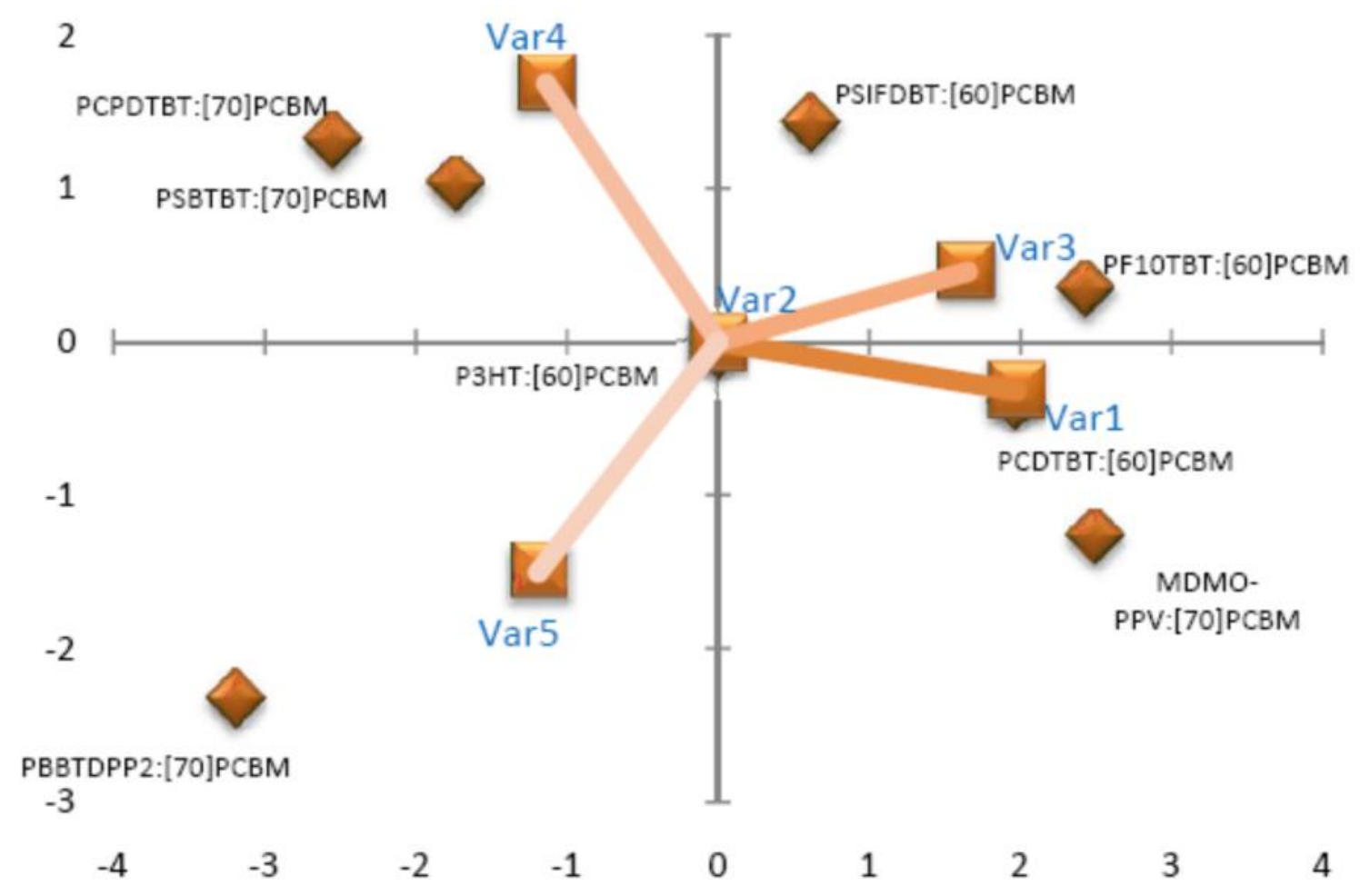

Fig.2. Déroulement d'une analyse en composantes principales.

Les carrés pour les variables, et les losanges pour les matériaux (observations)

Contributions des observations (\%) :

\begin{tabular}{lrrrr}
\hline & \multicolumn{1}{c}{$\mathrm{F} 1$} & \multicolumn{1}{c}{$\mathrm{F} 2$} & \multicolumn{1}{c}{$\mathrm{F} 3$} & \multicolumn{1}{c}{$\mathrm{F} 4$} \\
\hline PCPDTBT:[70]PCBM & 18,123 & 14,074 & 3,624 & 6,200 \\
PSIFDBT:[60]PCBM & 1,025 & 16,706 & 14,018 & 28,194 \\
PSBTBT:[70]PCBM & 8,416 & 8,470 & 1,041 & 13,569 \\
P3HT:[60]PCBM & 0,000 & 0,026 & 32,688 & 28,406 \\
PF10TBT:[60]PCBM & 16,294 & 0,879 & 19,070 & 0,113 \\
PBBTDPP2:[70]PCBM & 28,208 & 44,831 & 12,791 & 1,669 \\
PCDTBT:[60]PCBM & 10,666 & 1,253 & 0,882 & 21,176 \\
MDMO- & & & & \\
PPV:[70]PCBM & 17,269 & 13,759 & 15,885 & 0,671 \\
\hline
\end{tabular}

A partir de la figure 2 on peut remarquer qu'a partir d'une analyse ACP on peut avoir deux ensembles de données l'un regroupe la majorité des matériaux allié au PCBM (au $1^{\text {er }}$ et au $2^{\text {eme }}$ quadrant à base de C60 et le C70 pour les autres quadrants), et l'autre 
donne les variables données à la table 1 respectivement. De ces derniers en devine que le rendement d'une cellule solaire organique est inversement proportionnel à l'énergie de gap des matériaux donneurs.

\section{CONCLUSION}

Dans ce travail, nous avons présenté les résultats d'une étude d'analyse ACP pouvant conduire à la classification des paramètres caractérisant les cellules solaires organiques. Nous remarquons que les résultats obtenus par la méthode d'analyse en composante principale sont essentiellement qualitatifs. Les méthodes de classification automatique obligent à une précision rigoureuse dans le choix des variables prises en compte et des critères d'agrégation. S'il est vrai que l'analyse des données en général ne révèle que des évidences, cela est finalement heureux et permet d'autant plus d'intégrer cet outil dans la démarche de recherche.

\section{REFERENCES}

[1] M.A. Chikh, "Analyse du signal ECG par les réseaux de neurones et la logique floue :

Application à la reconnaissance des battements ventriculaires prématurés", Thèse présentée devant l'Université de Tlemcen, 2005.

[2] I.T. Jolliffe "Principal component analysis", Springer-Verlag, New York, 1986.

[3] K.I. Diamantaras, S.Y . Kung, "Principal component neural networks. Theory and applications", John Wiley and Sons , 1996.

[4] H. Hotelling "Analysis of a complex of statistical variables into principal components",

Journal of Educational Psychology, 24 (1933) 417- 441

[5] J. F. MacGregor , T. Kourti , "Statistical process control of multivariate process control"

Engineering Practice, 3, (1995) 403-414.

[6] C. Duby, S. Robin, "Analyse en Composantes Principales", Département O.M.I.P(2006).

[7] Lindsey Smith, " A tutorial on Principal Component Analysis», (2002).

[8] M. F. Harkat, "Détection et localisation de défauts par Analyse en Composantes

Principales ", Thèse de doctorat de l'Institut National Polytechnique de Lorraine (2003). 
[9] http://www.cs.otago.ac.nz/cosc453/student_tutorials/principal_components.pdf.

[10] S. Wold, K. Esbensen, P. Geladi , "Principal component analysis ", Chemometrics and Intelligent Laboratory Systems 2 (1987). 37-52

[11] P. Teppola, S. Mujunen, P. Minkkinen, T. Puijola, P. Pursiheimo "Principal component

component analysis", Chemometrics and Intelligent Laboratory Systems, 44 (1998) 307-317.

[12] L. Ferre, "Selection of components in principal component analysis : A comparaison

of methods", Computational Statistics and Data Analysis (1995) 669-682.

[13] D.Veldman, S.C.J.Meskers and A.J. Janssen Adv Funct. Mater. 2009 19? 19391948

[14] M. Benosman et B. Benyoucef , Calculs Hiérarchisés des Paramètres et Caractéristiques

Optimaux des Différents Matériaux Photovoltaïques 


\section{Figures}

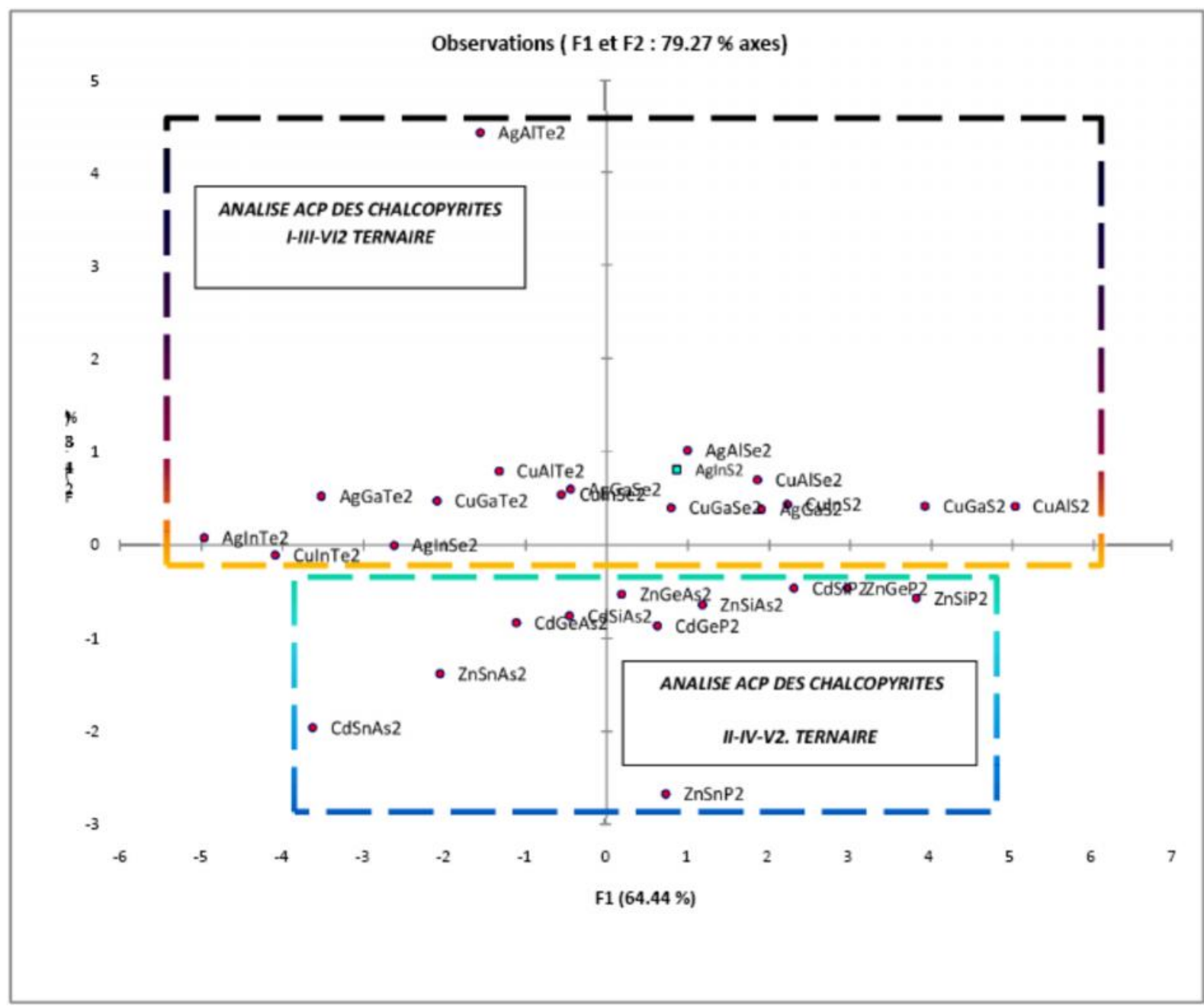

Figure.1 rassemblement générale et répartition des groupes de semi-conducteurs 


\section{ETUDE DES PERFORMANCES DES CELLULES SOLAIRES ORGANIQUES PAR UNE ANALYSE EN COMPOSANTES PRINCIPALES}

\section{RESUME}

L'analyse des données est actuellement, chez les statisticiens, l'objet d'un véritable phénomène de mode, caractérisé à la fois par l'engouement et le rejet. Déterminer les caractéristiques d'un ensemble de données n'est évidemment pas une fin en soi, mais cet oubli est malencontreux car le type de données disponibles influence toujours la direction de la recherche. L'analyse factorielle en composantes principales normées $(\mathrm{ACP})$, est particulièrement adaptée à la réduction de tableaux de mensurations, où l'on travaille le plus souvent sur une matrice de corrélation entre caractères. En cet article nous rapporterons nos résultats d'analyse des semi-conducteurs organiques en utilisant ce qu'on appel le datamining des matériaux. Toutes les informations ont été présentées dans les tables prévues par une méthode ACP des semi-conducteurs étudiés. L'analyse factorielle substitue aux mesures primitives de nouvelles variables ou facteurs (qui sont des combinaisons linéaires des variables primitives) à l'aide desquels il est donc possible d'optimiser la visualisation des données en un nombre restreint de figures. Gros au modo nous présentons, les résultats liés au développement de nouvelles approches du Datamining, l'analyse en composantes principales (ACP), des techniques informatiques qui ont été appliqué sur des semiconducteurs organiques et alliés avec des métaux pour prédire de nouveaux matériaux ou revêtements à hautes performances photovoltaïques.

Mots clés : cellules solaires organiques, l'énergie de gap, le rendement de conversion, $\mathrm{ACP}$

How to cite this article

Merad Boudia M R, Ferouani A M, Cheknane A and Benyoucef B. Study of performances of organic solar cells by analysis of main components. J Fundam Appl Sci. 2011, 3(1), 85-93. 